\title{
Archives of Palliative Care and Medicine
}

\section{A Storytelling Program for Women Living with HIV in an In- formal Settlement in Kenya: Addressing Psychological Trauma, Depression and Stigma}

\author{
Susan Njuguna ${ }^{1 *}$, Sylvia Tuikong ${ }^{2}$, Peter Ngure ${ }^{3}$ \\ ${ }^{1}$ Department of Nursing, Daystar University, Machakos, Kenya \\ ${ }^{2}$ Quality Assurance, Daystar University, Machakos, Kenya \\ ${ }^{3}$ Consortium for Advanced Research Training in Africa, Kenya
}

"Corresponding author: Susan Njuguna, Department of Nursing, Daystar University, Machakos, Kenya. Tel: +254722383401; Email: snjuguna@daystar.ac.ke

Citation: Njuguna S, Tuikong S, Ngure P (2018) A Storytelling Program for Women Living with HIV in an Informal Settlement in Kenya: Addressing Psychological Trauma, Depression and Stigma. Arch Palliat Care Med: APCM-108. DOI:10.29011/APCM-108.000008

Received Date: 21 July, 2018; Accepted Date: 17 September, 2018; Published Date: 24 September, 2018

\begin{abstract}
Awareness of HIV and AIDS in Kenya is high but stigma is still experienced among women living with HIV. The supportive environment in support groups is correlated with reducing apprehension and depression among people living with HIV although psychological issues are not adequately addressed. A randomized control study with the intervention of narrative approach was carried out for Women living with HIV. Eligibility included a score of above 21 on the Impact of Event (IES- R) Scale, severe depression (CES-D) and scored high on the Internal Stigma Scale (HIV ISS). A structured group therapy session using narrative approach was conducted weekly for twelve (12) weeks for five groups of ten (10) members each.

The finding was a reduction in the impact of event for all intervention groups on the Impact of Event Scale (IES-R). On the depressive scale, all members in the intervention groups moved from severe depression to moderate $37(75 \%)$ and mild $13(25 \%)$. The stigma scale indicated that $30(60 \%)$ of the participants improved to moderate and mild while 20(40\%) remained with severe stigma. Group therapy should be structured and adopt the narrative approach for women living with HIV. This will complement measures to reduce trauma from the impact of event, depression and internal stigma.
\end{abstract}

\section{Introduction}

The aspect of addressing feelings of isolation and other related emotional distress besides disclosure of HIV status forms the eligibility criteria for studies on psychosocial support for people living with HIV [1]. Psychological and emotional distress has an influence on the management of chronic illnesses as is seen in people living with HIV and may be unwell. HIV-status disclosure by people living with HIV and AIDS is widely advocated as a means to prevent HIV transmission [2]. This has received much attention in the international health literature $[3,4]$ On an individual level, disclosure facilitates people living with HIV to seek treatment and care and supports adherence to complex treatment regimens. Disclosure of one's status may also assist HIV prevention efforts in society as it enables others to learn from the experiences of people living with HIV [5] The World Health Organization (WHO) suggests that activities used in support groups for people living with HIV may help women develop the confidence to share their status with others [1]. A study from India found a higher rate of disclosure to partners by literate respondents compared with illiterate respondents [6]. Low-wage employment and economic vulnerability reduced disclosure by Tanzanian women [7]. In the last decade, there has been a dramatic rise in the number of HIV support groups in Kenya.

Despite these proposed benefits, many women living with HIV do not disclose their status for fear of blame, violence and abandonment by a partner, as well as stigma and isolation within the home and community [8]. Disclosure has significant health implications, firstly because the negative outcomes of disclosure 


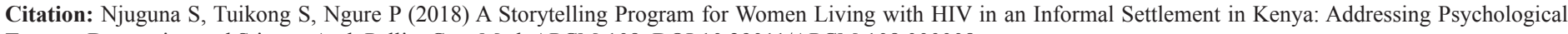
Trauma, Depression and Stigma. Arch Palliat Care Med: APCM-108. DOI:10.29011/APCM-108.000008

can be detrimental and severe for the women affected, and secondly because low rates of disclosure may increase cases of HIV transmission to others [9]. Narrative approach (also known as storytelling) in psychology, and in social science, explores the different stories told by the story teller to both provide insight into the actual character of the experience described and the insight it offers into the identity of the storyteller and of the culture in which she or he lives [10]. Narrative approach involves

Having conversation within a collaborative relationship where each member influences the understanding of the other by their contribution. The therapist and the person or group members both benefit from knowledge of the therapeutic interaction at the group session. The Kenya national HIV prevalence was estimated to be $5.6 \%$ among Kenyans aged $15-64$ years; this being a significant decline in rate of infection from previous estimates [11]. However, women aged 15-64 years still had higher HIV prevalence rates and young women aged20-24 years were at least three times more likely to be infected than young men of the same age group [11] Community level intervention should target women living with HIV among risk populations. HIV-prevention efforts are hindered by widespread poverty where almost $20 \%$ of people live on less than US\$ 1.25 a day [12].

Group level interventions in psychosocial support for people living with HIV have been shown to provide reduction in depression and anxiety [13]. This study describes an intervention at community level using the narrative approach that aimed at reducing the impact of trauma, depression and stigma among women living with HIV in an informal settlement in Kenya. HIV stigma experienced by people living with HIV can be enacted, anticipated or internalized. Enacted stigma involves experiences of discrimination, stereotyping and or prejudice from others due to one's HIV status. Anticipated stigma includes expectations of enacted stigma. Internalized stigma refers to a situation when stereotyping and or prejudice involving negative feelings and beliefs about people living with HIV.

\section{Materials and Methods}

This was a randomized control trial where the intervention used was the narrative approach (story telling) intervention for women living with HIV. The reference population was women living with HIV and attending an established on site services at public health centers in a sub county of Nairobi. Those recruited had been diagnosed within the last two years and were selected based on the scores for depression, impact of event and internal stigma scale of a five response items. This was based on the respondents perceived experiences for the past 7-day. Eligibility was based on the scores for Psychological distress above 21 on the psychological Impact of trauma IES-R (Impact of Event Scale-Revised) and depression index on CES-D (Center for Epidemiologic Studies-Depression) where clients met the criteria for major depressive episode (very severe) and probable major depressive (severe) episode based on DSM V; using 20-item CES-D [14,15] Despite the fact that the depressive index has been extensively used in studies on Mental health, there were very few studies on the use of the scale in people living with HIV. This informed the decision to adopt it for intervention for depression in people living with HIV. The 28item and internal stigma through ISS (Internal Stigma Scale) [16] were used to assess persons' internalized stigma from interacting with their community.

A study population of one hundred and twenty two clients meeting the above criteria was obtained, and an informed consent from each of them obtained before being assigned to the groups. Clients with the highest scores on the depressive index were conveniently selected to make up the five (5) intervention groups of ten members each; a total of fifty (50) members met weekly for the 12 weeks. The intervention groups were then assigned members based on age with the youngest 24-31being in group 1 and the oldest 46-55 being in group five. This was to allow participants to be as close as possible to the developmental stages of their group members. The remaining twenty eight (28) formed the control group that met monthly for the regular support group sessions with the community health volunteers. A Community health volunteer is the contact person between the community and the health system. They provide both preventive and promotive health services at community level and follow up the clients seen at the integrated site facilities. The support group addressed health and social issues without the narrative intervention. The members received medical attention on issues related to HIV and referrals made as appropriate.

\section{Procedure for Narrative Approach Group Session}

Each weekly group session lasting one hour at a time agreed upon by members, was facilitated by team leader who had been trained on the narrative approach as intervention. Of the five groups that were constituted, three groups were facilitated by professionals who included a Nurse, a Counselor and a Social worker. The other two groups were facilitated by Community health volunteers who were involved with counseling of women and girls living with HIV through a community based organization. All the team leaders had basic counseling skills and were practicing professionals. Each of the five groups had two (2) Community health volunteers who participated by observing in the intervention group and also facilitated the monthly meetings of four support groups (the control group) with seven members each. The community health volunteers on the special intervention group were excluded from the support group as they were already involved with younger women requiring crisis therapy and support.

The inclusion of Community health volunteers was to contribute towards mentorship into the narrative approach and for 


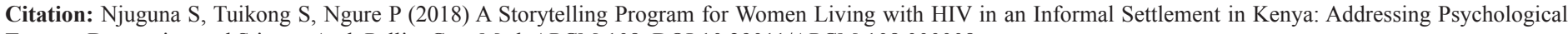
Trauma, Depression and Stigma. Arch Palliat Care Med: APCM-108. DOI:10.29011/APCM-108.000008

continuity beyond the stipulated project period. The community health volunteers administered the questionnaires to all group members after the four week sessions. This was intentional to minimize bias which would have been the case if the team leaders who facilitated the sessions administered the questionnaires. Data was collected using self administered questionnaires from all groups (one hundred and twenty two (122) clients) every four weeks using the survey tool where participants responded to the questions based on the immediate past 7 days. Quantitative data collected was analyzed using the established scale.

A standardized structured guideline with specific objectives for each session was used by all groups for each of the twelve (12) weeks. All the groups got the same intervention. The participants in each group were required to relate to and have input from other group members on possible solutions to the issues related to impact of illness, depression and internal stigma. The discussions addressed challenges faced by the women and how they coped, their perception of how HIV altered their lives, the psychosocial and health needs that they faced. Each group member was given an assignment after the sessions that required writing or drawing relating to the events that had been discussed. The team leader was able to pick out cues to improvement or need for individual counseling from the assignments. Individual psychological counseling was provided to clients from both groups who required it. Each team leader and community health volunteers observed verbal and non- verbal communication of members and responded appropriately.

Data from the survey completed by each participant was analyzed using guideline for analysis and interpretation of the scales for Trauma, IES-R (Impact of Event - Revised); Depression, CES-D (Center for Epidemiological, Studies Depression) and HIV Stigma: ISS (Internal Stigma Scale). The findings are summarized below.

\section{Results and Discussion}

\section{General Information}

Participants completed a questionnaire that provided information on the demographics (age, education status, employment status, marital status, the number of family member, experience of losing their child (ren), and income), social support. The mean age for the participants was 39.25 with a standard deviation of 8.49 and a range of 24- 60 years. All the participants were low income earners $122(100 \%)$. Those that were married and living with spouse or having been widowed were 74(60.7\%). Complex trauma associated with other incidences like loss of a child or spouse may further complicate the management of women living with HIV.

\section{Psychological Status}

The baseline findings on trauma, depression and internal stigma are presented in Table 1 above. Those recruited into the sample had a score of 21 and above on Impact of traumatic Experience (IES-R.) representing a mean score of 53.26. There were $78(64 \%)$ of those selected who had severe and very severe depression. Fifty (50) members with very severe depression were assigned to five (5) intervention groups of ten members each. The twenty eight (28) who had severe depression and were not included in the intervention group were taken through support group therapy and individual counseling as was appropriate. The findings from the control group indicated very minimal change on all the scales under investigation. This may be explained by the fact that the support group session though being managed by the community health volunteers were not structured to produce definite results. Following the twelve week narrative approach intervention with the intervention group sessions, the findings were as follows:

\section{Trauma (Impact of Traumatic event)}

The surveys were carried out at baseline and every four weeks for a total of four surveys. The assessment for Trauma (IES-R) was based on the scale as mild 0-20; moderate 21-32; severe 33- 44, very severe $45-88$. There was a general reduction in the impact of event with major improvement being seen in groups 1,2, 3 and 5 as shown in Table 1 below.

\begin{tabular}{|c|c|c|c|c|c|c|}
\hline Survey & \multicolumn{7}{|c|}{ Group Number (age bracket) } \\
\hline Week & & $1(24-31)$ & $2(48-55)$ & $3(39-45)$ & 4 (special mixed) & $5(32-38)$ \\
\hline \multirow{3}{*}{ IES-R } & 1 & 49.6 & 55.25 & 59.13 & 74.63 & 54.17 \\
\cline { 2 - 7 } & 2 & 46.9 & 30.38 & 51 & 27.38 & 43.5 \\
\cline { 2 - 7 } & 3 & 40.4 & 31.71 & 45.83 & 52.88 & 51.33 \\
\cline { 2 - 7 } & 4 & 34.7 & 20.5 & 37.25 & 65 & 24.33 \\
\hline
\end{tabular}

Table 1: Trauma (IES-R). 


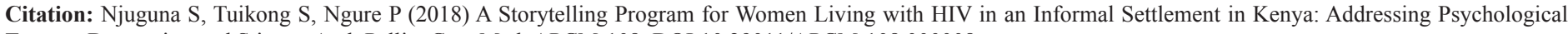
Trauma, Depression and Stigma. Arch Palliat Care Med: APCM-108. DOI:10.29011/APCM-108.000008

The perception of impact of trauma reported based on the past seven days by participants of each group indicated mean changes across all age groups with the older members (48-55 years) having the greatest improvement.

\section{Depression}

The members of the intervention group were conveniently sampled based on the depressive scale measure of severe depression. Following the intervention there was marked improvement across all age groups as in Table 2 .

\begin{tabular}{|c|c|c|c|c|c|c|}
\hline \multicolumn{2}{|c|}{ Survey Weeks } & \multicolumn{5}{|c|}{ Group Number (age bracket) } \\
\hline & & $1(24-31)$ & $2(48-55)$ & $3(39-45)$ & $4($ special mixed) & $5(32-38)$ \\
\hline & 1 & 38 & 37.5 & 42 & 46.3 & 41.7 \\
\hline CES-D & 2 & 24.9 & 21.8 & 32.1 & 22.4 & 35.9 \\
\hline & 3 & 21.6 & 18.3 & 26.2 & 30.7 & 32.4 \\
\hline
\end{tabular}

Table 2: Depression (CES-D).

On the depressive scale, all members from the intervention groups moved from severe depression at the baseline survey to moderate $37(75 \%)$ and mild $13(25 \%)$ at week four survey.

\section{Internal Stigma}

Perceived, internalized HIV stigma was prevalent among participants. On the Internal stigma scale, the findings did indicate some improvement.

\begin{tabular}{|c|c|c|c|c|c|c|}
\hline Survey Weeks & \multicolumn{5}{|c|}{ Group Number (age bracket) } \\
\hline & & $1(24-31)$ & $2(48-55)$ & $3(39-45)$ & $4($ special mixed) & $5(32-38)$ \\
\hline & 1 & 63.1 & 60.3 & 65.3 & 79.4 & 66.7 \\
\hline Stigma & 2 & 34 & 41.4 & 66.1 & 66.6 & 68.5 \\
\hline & 3 & 32.5 & 35.1 & 47.7 & 53.3 \\
\hline
\end{tabular}

Table 3: Stigma (ISS).

However, $40 \%$ of the intervention group members remained at severe on ISS with group four members showing worse results at the twelve-week survey compared to the baseline.

\section{Discussion}

The study aimed to present the narrative approach as an intervention that can contribute to the reduction of the impact of trauma, depression and stigma among women living with HIV and AIDS in the informal settlement in Kenya. These are preliminary findings of work in progress.

The results indicate that the impact of events for the participants (IES-R) improved from very severe $(100 \%)$ for all groups to severe (40\%) moderate (40\%) and mild $(20 \%)$. The findings concur with assertions from other studies [17]. The disruption and fragmentation manifested in traumatizing experiences serves as a useful means of highlighting the sense of unity, meaning and coherence (the 'narrative configuration') more commonly experienced on an everyday level. When disorder and incoherence prevail, as in the case of trauma, narratives are used to rebuild the individual's shattered sense of identity and meaning. The sustained weekly sessions where participants were able to review the traumatizing events of their lives in group session may have contributed to this outcome.

CES-D depressive level changed from $100 \%$ (very severe and severe) to moderate (75\%) and mild (25). The findings are in agreement with other studies where psychotherapy was found to reduce the depressive levels. Regardless of study designs, all studies reported positive intervention effects, ranging from a reduction in HIV and AIDS stigma, loneliness, marginalization, distress, depression, anger, and anxiety to an increase in selfesteem, self- efficacy, coping skills and quality of life [18].

ISS findings indicated that $60 \%$ of the women moved to moderate from very severe, with $40 \%$ remaining at very severe. This could be possibly explained by the fact that this was a mixed age's group where participants probably faced trauma differently. Stigma is a complex issue that requires to be contextualized for better understanding [19]. Deacon [19], suggests limiting the definition of stigma to the process of othering, blaming 


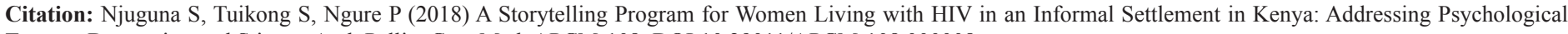
Trauma, Depression and Stigma. Arch Palliat Care Med: APCM-108. DOI:10.29011/APCM-108.000008

and shaming (often called symbolic stigma). She suggests that analytically separating stigma from discrimination would lead to a better understanding of the relationship between them and that there is need to understand discrimination caused by stigma as only one element of stigma. The findings are in agreement with other studies which found that perceived and internalized HIV stigma were prevalent among participants, and resulted in various burdens with different magnitudes based on their life contexts.

\section{Study Limitations}

The results cannot be generalized to the entire population of people living with HIV in Kenya but provides useful insights on psychological support for women living with HIV. All variables were measured by self-reporting instruments, which may have caused some bias. It is important for future studies also to use other forms of data collection, for example expert judgment. Other aspects that could influence the psychological well-being, such as coping strategies were not included in the study. Future studies should address these other aspects.

\section{Conclusion/Recommendation}

Psychological and social support is necessary to address the impact of HIV. Women living with HIV can benefit from interventions like the narrative approach besides other interventions to Especially address psychological issues like depression to complement social and medical support which is already a part of the management of people living with HIV. Psychological support goes a long way in reducing the impact of trauma, depression and stigma associated with HIV. Inclusion of the narrative approach in the therapy of people living with HIV/ AIDs is recommended as providing long term positive effects in reducing stigma both in newly diagnosed clients and those previously diagnosed. Organizations working with people living with HIV and AIDs should consider inclusion of the narrative approach within the contexts of the communities that they work with to complement measures aimed at reducing stigma and trauma.

\section{Ethics Approval}

Ethics approval for the study was obtained from the Kenya National AIDS Council, the Director, Nairobi County Health services before the launch of the study. Consent was obtained from and each participant, before recruitment and before every survey throughout the study period.

\section{Competing Interests}

The authors declare no competing interests.

\section{Authors' Contributions}

All authors have contributed to this study in ways consistent with the authorship criteria. All authors read and approved the final manuscript.

\section{Acknowledgments}

We wish to acknowledge Jeong Ryu, Yonsei University, Korea. Namhee Choi, Seoul Institute for Narrative Studies, Korea and Seoul Women's College of Nursing, Korea, for their contribution. SINAS- Korea, Daystar University, DASCO and Tuinuke for their support.

\section{References}

1. WHO (2009) A Qualitative review of psychosocial support for young people living with HIV. 36.

2. UNAIDS (2000) Opening up the AIDS Epidemic: Guidance on Encouraging Beneficial Disclosure, Ethical Partner Counseling and Appropriate Use of HIV case- Reporting.40.

3. Maman S, Mbwambo J, Hogan N M, Kilonzo G P\& Sweat M (2001) Women's barriers to HIV-1 testing and disclosure: ch allenges for HIV-1 voluntary counseling and testing. AIDS Care 13: 595-603.

4. Medley A, Garcia-Moreno C, McGill S, Maman S (2004) Rates, barriers and outcomes of HIV-serostatus disclosure among women in developing countries: Implications for prevention-of-mother-to-childtransmission programs. Bulletin of the World Health Organization 82: 299-307.

5. Paxton S. (2002) Impact of Utilizing HIV-Positive Speakers in AIDS Education. AIDS Ed \& Prev 14: 282-294.

6. Taraphdar P, Dasgupta A, Saha B (2007) Disclosure among people living with AIDS. Indian J Community Med 32: 280-282.

7. Antelman G, Smith Fawzi MC, Kaaya S (2001) Predictors of HIV-1 serostatus disclosure: a prospective study among HIV-infected pregnant women in Dar es Salaam, Tanzania. AIDS 15: 1865-1874.

8. Greeff M, Phetlhu R, Makoae LN, Dlamini PS, Holzemer WL, et al. (2008) Disclosure of HIV status: experiences and Perceptions of persons living with AIDS and nurses involved in their care in Africa. Qualitative Health Research 18: 311-324.

9. UNAIDS (2006) Report on the Global AIDS Epidemic. Geneva.

10. Murray M (1997) A Narrative Approach to Health Psychology Background and Potential. J Health Psycho 2: 9-20.

11. KAIS (2012) Kenya AIDS Indicator Survey 2012.

12. UNDP (2010) Human Development Report 2010.

13. Hyde J, Appleby PR, Weiss G, Bailey J, Morgan X (2005) Group-level interventions for persons living with HIV: a catalyst for individual change. AIDS Educ Prev 17: 53-65.

14. Radloff LS (1977) The CES-D: A self-report depression scale for research in the general population. Applied psychological measurement 3: 385-401.

15. Rudolf LS, Terry L (1986) Use of the center for epidemiological studiesdepression scale with older adults. Clinical Gerontologist 5: 119-136.

16. Sowell RL, Lowenstein A, Moneyham L, Demi A, Mizuno $Y$, et al. (1997) Resources stigma and patterns of Disclosure in Rural Women with HIV infection. Public Health Nursing 14: 302-312. 
Citation: Njuguna S, Tuikong S, Ngure P (2018) A Storytelling Program for Women Living with HIV in an Informal Settlement in Kenya: Addressing Psychological Trauma, Depression and Stigma. Arch Palliat Care Med: APCM-108. DOI:10.29011/APCM-108.000008

17. Croslley L, Michele (2000) Narrative Psychology, Trauma and the study of Theory Psychology 10: 527-546.

18. Wu L (2013) Community-based AIDS STAGE interventions to promote psychosocial well-being among people living with AIDS STAGE: a literature review. Health Psychol Behav Med 1: 31-46.
19. Deacon $\mathrm{H}$ (2006) Towards a sustainable theory of Health-related stigma: Lessons from the AIDS literature. Journal of Community and applied Social Psychology 16: 418-425. 